\title{
Epitaxial growth of GaAs by solid-phase transport
}

\author{
J. S. Chen, E. Kolawa, C. M. Garland, and M-A. Nicolet \\ California Institute of Technology, Pasadena, California 91125
}

(Received 8 April 1991; accepted for publication 1 July 1991)

(100) GaAs substrates with an Ag film about $45 \mathrm{~nm}$ thick were first annealed at $550{ }^{\circ} \mathrm{C}$ for

$30 \mathrm{~min}$ in an Ar-flowing furnace (preannealing). A 110-nm-thick GaAs layer was then

deposited on top of the preannealed $\langle\mathrm{GaAs}\rangle / \mathrm{Ag}$ samples, followed by an amorphous Ta-Si-N

film that was deposited over the GaAs layer to serve as a cap layer to minimize the loss

of As during the following annealing process. The completed structures were then annealed

again at $550^{\circ} \mathrm{C}$ for $30 \mathrm{~min}$ in flowing Ar. The transport of $\mathrm{Ga}$ and As through the $\mathrm{Ag}$

layer and an epitaxial growth of GaAs on top of the (100) GaAs substrate are observed by

cross-sectional transmission electron microscopy and $\mathrm{MeV}{ }^{4} \mathrm{He}$ backscattering

spectrometry. No GaAs epitaxial growth is observed in samples that are not preannealed.

Our results demonstrate that epigrowth through a solid transport medium is possible

for a III-V semiconductor as it is for $\mathrm{Si}$ and $\mathrm{Ge}$.

Solid-phase epitaxy, either with or without transport medium (a metal layer, usually), is well documented for $\mathrm{Si}$ and $\mathrm{Ge}^{\prime}{ }^{\prime}$ In contrast, solid-phase epitaxy of compound semiconductors is a recent discovery. The solid-phase regrowth of doped (or alloyed) GaAs layers by reactiondriven decomposition of intermediate phases has been documented in connection with the fabrication of ohmic contacts of $n$-type GaAs ${ }^{2-5}$ The sample configuration used in this type of solid-phase regrowth can be represented by GaAs (substrate) $/ M / X$. GaAs from the substrate is first consumed by reacting it with the intermediate metal layer $M$ at relatively low temperatures. The structure is then annealed at a higher temperature. The preferential reactions between elements of $X$ and $M$ then induce a precipitation and growth of $X$-doped GaAs or of a $\mathrm{Ga}_{y} \mathrm{X}_{1-y} \mathrm{As}$ alloy onto the substrate by solid-phase reaction and transport.

In the present study, we demonstrate the existence of solid-phase epitaxy of GaAs by transport of GaAs source material through a solid metal medium onto a GaAs single-crystal substrate, in exact analogy with the classical $\mathrm{Si}$ and Ge experiments. ${ }^{1}$ The first problem encountered is that of a suitable transport medium. The simplest choice is an element which has a tieline with GaAs in the corresponding ternary phase diagram. This is the only type of elemental transport medium that will be in thermodynamic equilibrium with $\mathrm{GaAs}$ in a closed system. ${ }^{6} \mathrm{Si}, \mathrm{Ge}, \mathrm{Re}, \mathrm{Ag}, \mathrm{Au}$, $\mathrm{Sn}$, and $\mathrm{W}$ belong to this category of elements. ${ }^{6,7} \mathrm{We}$ chose $\mathrm{Ag}$ as the transport medium because it has the largest solid solubilities for $\mathrm{Ga}$ and $\mathrm{As},{ }^{8}$ although we recognize that it is the high diffusivities through the transport medium for both constituent species that form the primary prerequisite for solid-phase growth of a binary compound.

A semi-insulating (100) GaAs substrate was degreased and chemically etched in $\mathrm{HCl}: \mathrm{H}_{2} \mathrm{O}$ (1:1 by volume) prior to the deposition of an $\mathrm{Ag}$ film about $45 \mathrm{~nm}$ thick by $e$-beam evaporation at a base pressure of $10^{-8}$ Torr. Under these conditions, a native oxide layer between the GaAs substrate and as-deposited $\mathrm{Ag}$ layer is inevitable. The native oxide layer interferes with the solid-phase epitaxy. To solve this problem, the $\langle\mathrm{GaAs}\rangle / \mathrm{Ag}$ structures were prean- nealed at $550{ }^{\circ} \mathrm{C}$ for $30 \mathrm{~min}$ in a flowing Ar furnace. (A parallel experiment omitting the preannealing step was done for comparison; no epitaxial growth was observed in those samples, see below.) A 110-nm-thick GaAs layer was then deposited by dc sputtering on top of the $\langle\mathrm{GaAs}\rangle / \mathrm{Ag}$ samples. Subsequently, an amorphous Ta-Si-N film was deposited over the GaAs layer by rf sputtering in yet another deposition system. This layer serves as a cap to minimize the loss of As during the flowing annealing process. An amorphous Ta-Si-N film has been proven to be a good encapsulating layer for GaAs. ${ }^{9}$ The completed structures were then again annealed at $550{ }^{\circ} \mathrm{C}$ for $30 \mathrm{~min}$ in an $\mathrm{Ar}$ flow. Samples were characterized by $\mathrm{MeV}^{4} \mathrm{He}$ backscattering spectrometry and transmission electron microscopy on cross-sectional samples using a Philips EM 430 microscope operating at $300 \mathrm{keV}$.

Figure 1 shows the backscattering spectra of completed structures ( $\langle\mathrm{GaAs}\rangle / \mathrm{Ag} / \mathrm{GaAs} / \mathrm{Ta}-\mathrm{Si}-\mathrm{N})$ with preannealed $\langle\mathrm{GaAs}\rangle / \mathrm{Ag}$ before and after the second annealing treatment. The height of the GaAs substrate signal increases after the second annealing step. This indicates

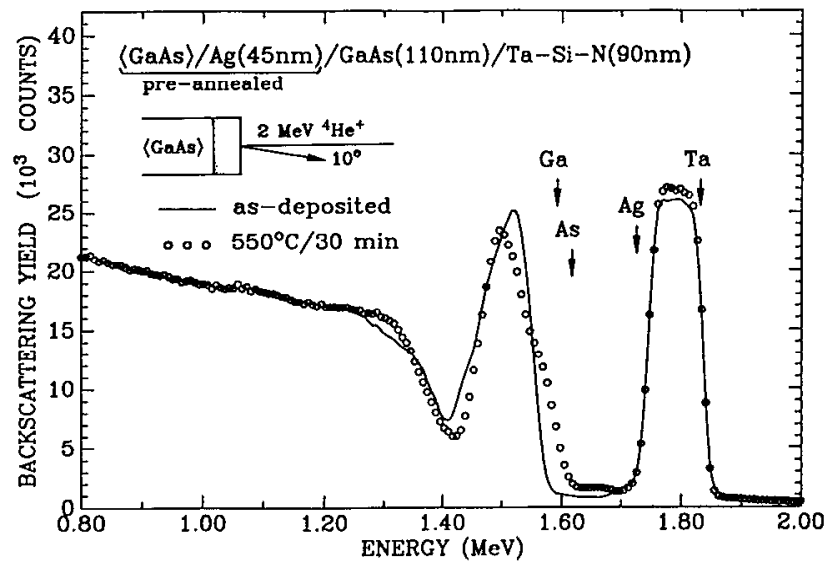

FIG. $1.2 \mathrm{MeV}^{4} \mathrm{He}^{+}$backscattering spectra of samples with the structure (GaAs)/Ag/GaAs/Ta-Si-N before and after annealing. The $\langle\mathrm{GaAs}\rangle / \mathrm{Ag}$ structure had been preannealed $\left(550^{\circ} \mathrm{C} / 30 \mathrm{~min}\right)$ prior to the deposition of the GaAs and Ta-Si-N layers. 


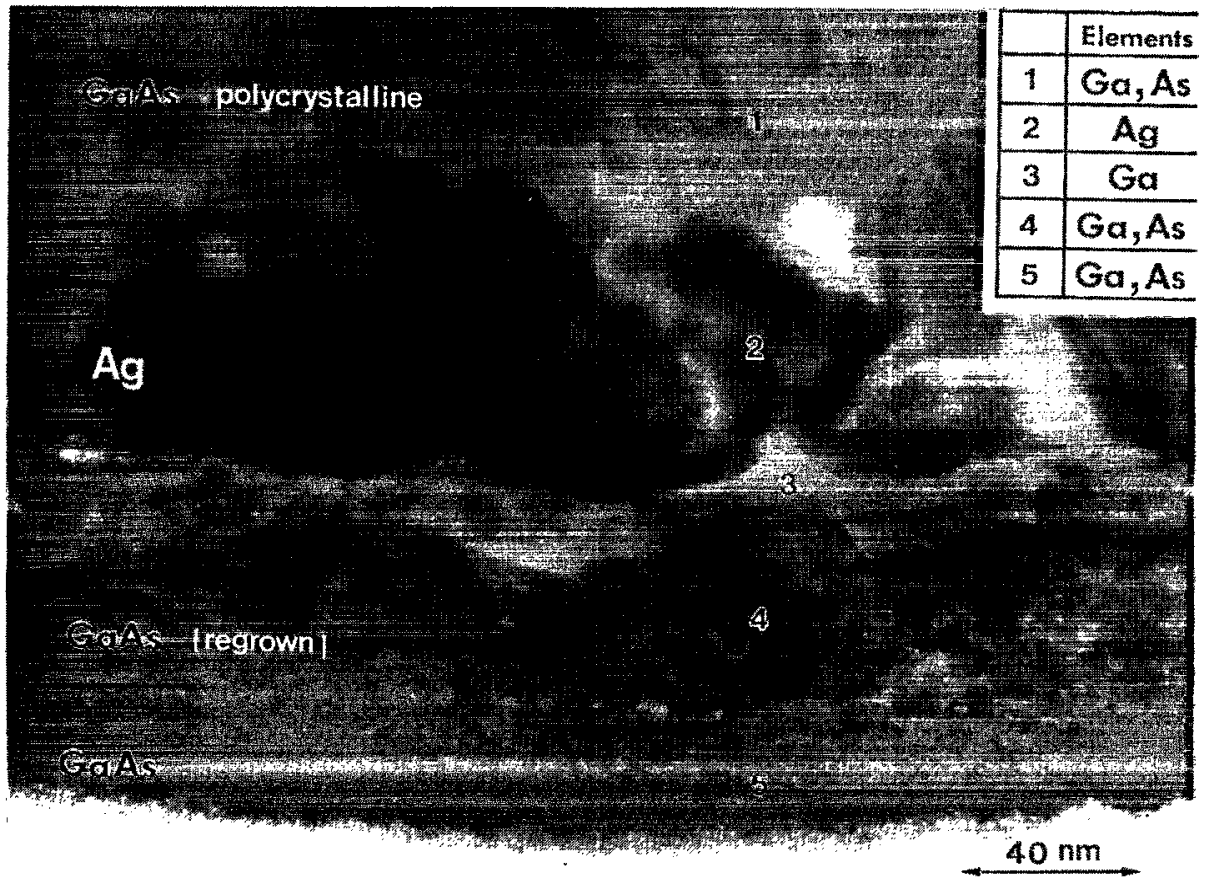

FIG. 2. Cross-sectional transmission elec

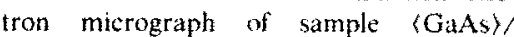
$\Lambda \mathrm{g}$ (preannealed)/GaAs/Ta-Si-N after annealing for $30 \mathrm{~min}$ at $550^{\circ} \mathrm{C}$. The epigrown GaAs layer is separated from the substrate by a white spot line. The elemental composition at position $1-5$ as determined by en. ergy-dispersive analysis of $x$-rays is tabulated on the upper right. that there is growth of GaAs on the substrate by solidphase reaction. In contrast, without preannealing of the 〈GaAs〉/Ag structure, there is no change in backscattering spectrum after annealing for the completed structures. It is apparent that the preannealing is a necessary step to achieve solid-phase epitaxial growth.

Cross-sectional micrographs taken on a as-prepared preannealed sample show that at that stage the deposited GaAs layer is polycrystalline. After the second annealing, cross-sectional micrographs reveal the existence of GaAs solid-phase epitaxy (Fig. 2). The sample was first investi- gated by energy-dispersive analysis of $x$-rays in the scanning transmission electron microscopy mode to obtain the depth distribution of elements. The results are tabulated in the insert of Fig. 2. Within the sensitivity limit of this technique (5 wt \%), no $\mathrm{Ga}$ or $\mathrm{As}$ is detected in the $\mathrm{Ag}$ layer, and vice versa. Selected area diffraction of the GaAs region below the Ag shows a single-crystal pattern. Nevertheless, this area is divided into two subregions by a line of fine white spots. This line marks the interface between the GaAs substrate and the epitaxially grown GaAs layer. The evidence is given in Fig. 3 which is a (200) dark-field

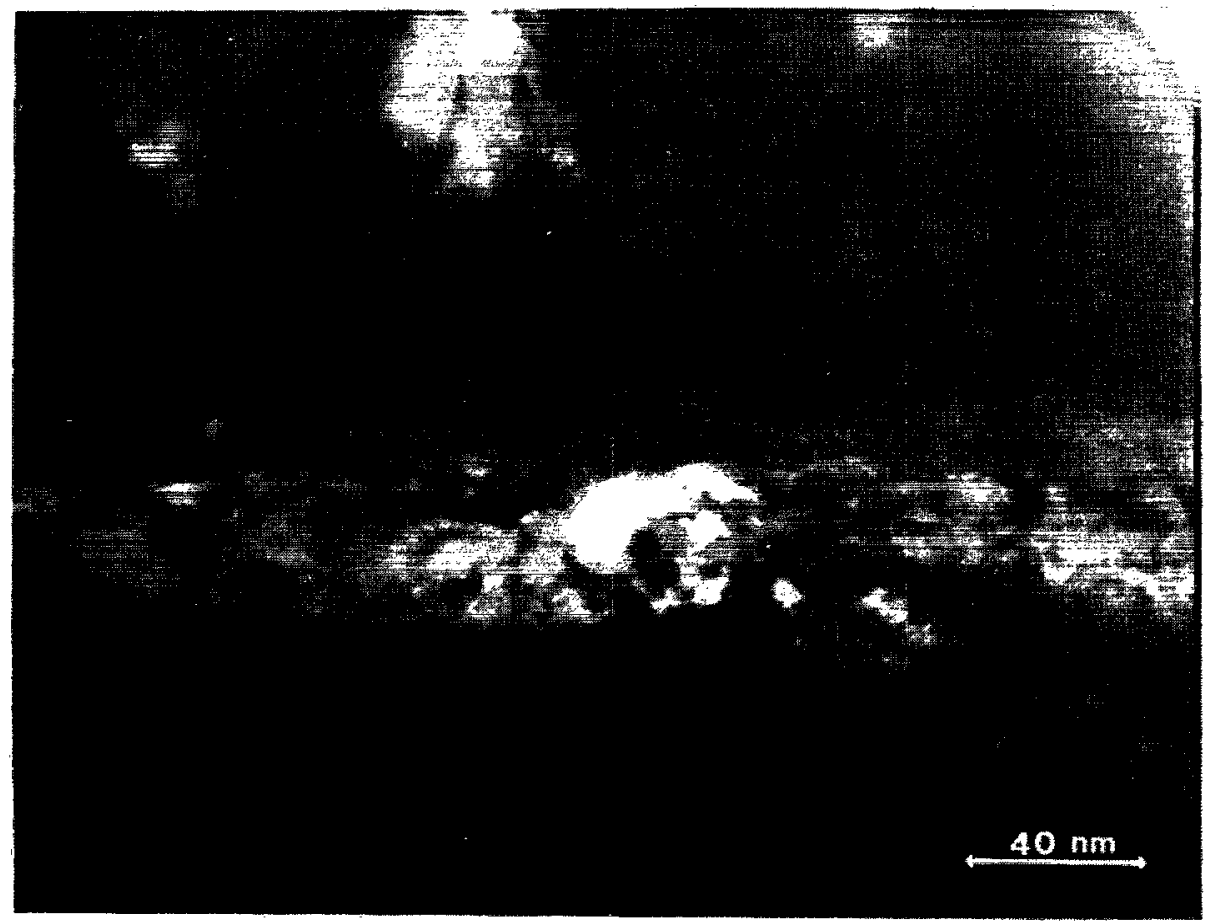

FIG. 3. (200) dark-field transmission electron micrograph of the same region shown in Fig. 2. The epigrown region is highlighted against that of the substrate due to the dif ferent deviation parameters induced by lat tice strains in the epigrown layer. 


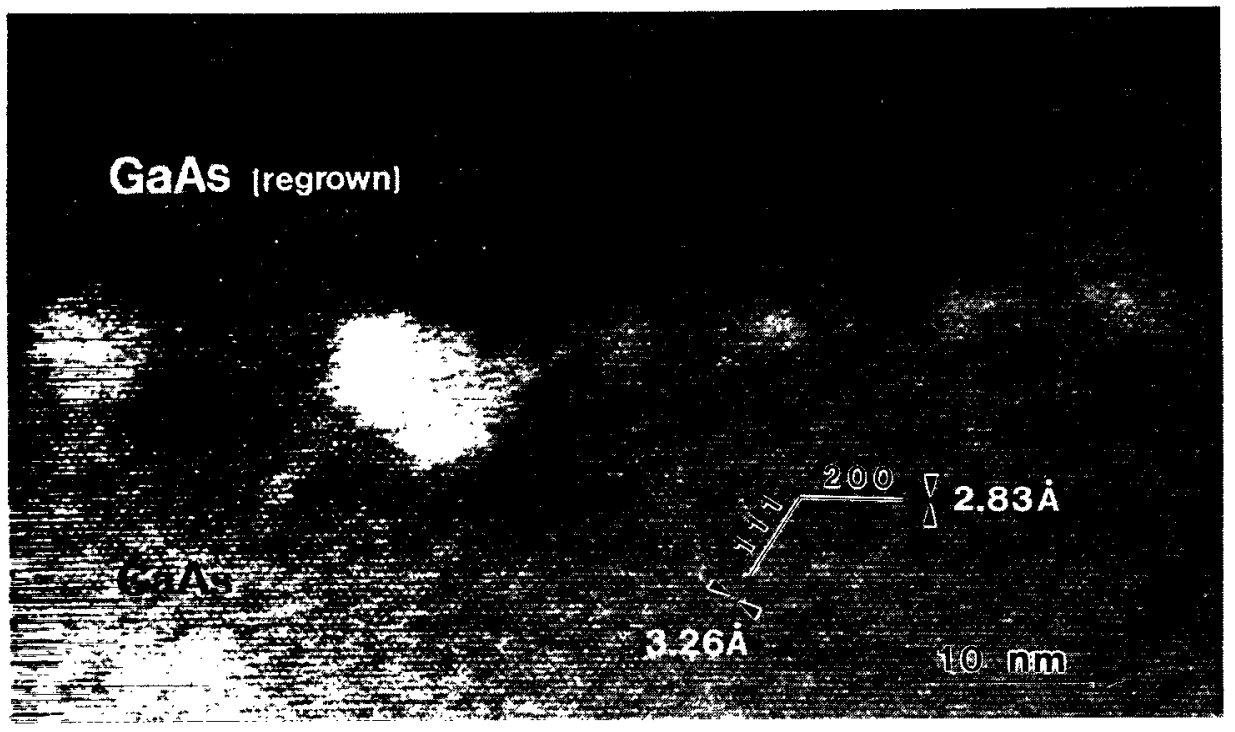

FIG. 4. High-resolution cross-sectional transmission electron micrograph of interface between the regrown GaAs layer and the GaAs substrate. The large white patch shows contrast of amorphous structure. It probably consists of native oxide and marks the location of the original substrate surface.

image of the same region shown in bright field in Fig. 2. Using the (200) diffraction beam, because of the different deviation parameter arising from the defect-induced strains or slight misorientation of the epigrown layer, the epigrown region is highlighted against that of the substrate.

High-resolution cross-sectional transmission electron micrography was employed to examine in detail the interfacial microstructure between the grown GaAs layer and the substrate. The lattice image of GaAs is continuous from the substrate through the grown layer (Fig. 4), but the epilayer is not perfect. Some fringes are blocked by white patches (the white spots in Fig. 2). The contrast of those patches reveals an amorphous internal structure. We believe that they are made of the native oxide originally located between the GaAs substrate and the deposited $\mathrm{Ag}$ layer. From a parallel study of the reactions that take place in a $\langle\mathrm{GaAs}\rangle / \mathrm{Ag}$ sample, we know that this native oxide balls up during the preannealing step and uncovers oxidefree regions. ${ }^{10}$ This scenario explains the need for a preannealing step, the presence of the white spots in Fig. 2, and warrants their interpretation as markers that indicate the position of the original substrate surface. We therefore predict that on an oxide-free surface, solid-phase epitaxy of GaAs through a transport medium should be straightforward to accomplish in a single annealing step. This discovery is potentially important for device application. Generalizing further, we expect solid-pahse epitaxy is possible quite generally for binary semiconductors providing that a suitable transport medium exists.

A distinct layer exists between the epigrown GaAs and the Ag layers (see Fig. 2). Gallium is the only element detected by energy dispersive analysis of $x$-rays in this layer. In some of these regions, lattice fringes corresponding to (002) and (113) planes of $\gamma-\mathrm{Ga}_{2} \mathrm{O}_{3}$ have been observed, indicating that the layer consists of gallium oxide. The origin of this layer and its location are being investigated.

This work was supported by the Army Research Office under a contract aiming at the development of stable contacts to semiconductors. The authors wish to thank Dr. Simon C. W. Nieh for valuable advice in transmission electron microscopy work and helpful discussions.

'S. S. Lau and W. F. van der Weg, in Thin Films-Interdiffusion and Reactions, edited by J. M. Poate, K. N. Tu, and J. W. Mayer (Wiley, New York, 1978), p. 433.

${ }^{2}$ E. D. Marshall, W. X. Chen, C. S. Wu, S. S. Lau, and T. F. Kuech, Appl. Phys. Lett. 47, 298 (1985)

${ }^{3}$ L. C. Wang, B. Zhang, F. Fang, E. D. Marshall, S. S. Lau, T. Sands, and T. F. Kuech, J. Mater. Res. 3, 922 (1988).

${ }^{+}$T. Sands, E. D. Marshall, and L. C. Wang, J. Mater. Res. 3, 914 (1988).

${ }^{5}$ T. Sands, J. P. Harbison, W. K. Chan, S. A. Schwarz, C. C. Chang, C. J. Palmstrom, and V. G. Keramidas, Appl. Phys. Lett. 52, 1216 (1988).

${ }^{6}$ R. Beyers, Ki Bum Kim, and R. Sinclair, J. Appl. Phys. 61, 2195 (1987).

'R. Schmid-Fetzcr, J. Elcetronic Mater. 17, 193 (1988).

${ }^{8}$ Binary Alloy Phase Diagrams, 2nd ed., edited by T. B. Massalski (ASM International, Metals Park, Ohio, 1990).

${ }^{\circ} J$, M. Molarius, E. Kolawa, K. Morishita, M-A. Nicolet, J. L. Tandon, J. A. Leavitt, and L. C. McIntyre, Jr., J. Electrochem. Soc. 138, 834 (1991).

${ }^{10}$ J. S. Chen, E. Kolawa, M-A. Nicolet, and R. P. Ruiz, MRS Spring Meeting, 1991 (to be published). 